\title{
ESTERASE VARIABILITY IN THE POPULATIONS OF CULEX QUINQUEFASCIATUS SAY 1823 FROM TWO GEOGRAPHICALLY ISOLATED LOCATIONS IN BANGLADESH
}

\author{
Reza Md. Shahjahan*, Rowshan Ara Begum, Marufa Rumman, \\ Farhana Sultana Karim and Anwara Begum \\ Laboratory of Animal Genetics \& Molecular Biology, Department of Zoology, \\ University of Dhaka, Dhaka-1000, Bangladesh
}

Key words: Esterase variability, Culex quinquefasciatus, Isolated locations

\begin{abstract}
Indiscriminate and frequent use of insecticides are causing occurrence of resistance problem in the treated population in mosquitoes. Organophosphates are associated with esterase enzyme overproduction leading towards the selection of resistant population. Esterase pattern was studied from two geographically isolated populations of Culex quinquefasciatus by the Polyacrylamide Gel Electrophoresis (PAGE). The municipal area of Sirajganj $\left(24^{\circ} 19^{\prime} 48^{\prime \prime} \mathrm{N}, 8^{\circ} 37^{\prime} 12^{\prime \prime} \mathrm{E}\right)$ is located $160 \mathrm{~km}$ away from Dhaka $\left(23^{\circ} 42^{\prime} 0^{\prime \prime} \mathrm{N}\right.$, $90^{\circ} 22^{\prime} 30^{\prime \prime E}$ ), the capital of Bangladesh. In Dhaka insecticides are frequently sprayed with by the city corporation whereas in Sirajganj town insecticides usually not in use. Altogether eight and seven esterase bands were observed from above two populations. These were named as Est-1, Est-2, Est-3, Est-4, Est-5 Est-6, Est-7, Est-8, respectively. Sirajganj population in general showed less esterase activity in comparison with that of Hatirjheel. Est-5 in Hatirjheel population was found to be distinct and darkly stained. These reflect increasing insecticide resistance due to excessive use of insecticides in mosquito control program in the Dhaka city. Furthermore, in Sirajganj population two female samples were found to produce unique bands in Est-2, very thick ( 50-100fold), although these specimens were morphologically similar to other samples, alleged to be the occurrence of species complex in Culex quinquefasciatus. Nation wide intensive sampling and large scale study in terms of esterase appears to be essential to reveal the entity of species complex and correlate resistance with the esterases for managerial purpose.
\end{abstract}

\section{Introduction}

Mosquitoes are insects those belong to the order Diptera. Over 2500 different species of mosquitoes have been reported world-wide and of $113 \mathrm{spp}$. have in Bangladesh.(1) Culex quinquefasciatus is one of the most nuisance species. It is annoying that cause serious problem in human health and domestic animals. They are capable of transmitting diseases such as filariasis and encephalitis to humans and animals. ${ }^{(1)}$

*Corresponding author: <dr_reza_111@yahoo.com> 
The enhancement of disease transmission is done because of the process of rapid urbanization and unplanned growth of cities. These have resulted into man made mosquito proliferation habitats promoting the breeding of a variety of disease vectors. Lack of adequate drainage and in many areas, even the provision of drainage, and water stagnation is promoting the breeding of $C x$. quinquefasciatus and the spread of filariasis due to Wuchereria bancrofti. ${ }^{(2)}$

Several studies have been conducted in different regions of the United States on populations of $C x$. p. pipiens complex where both subspecies and their intermediate forms occur. (3-4) These studies were conducted primarily to demonstrate genetic and morphological changes that occur in the transition zone from $C x$. p. pipiens to $C x$. $p$. quinquefasciatus. The main criteria used were difference in the allozyme variation. ${ }^{5-}$ 7) In Bangladesh, population genetics studies on $C x$. quinquefasciatus have not yet been conducted.

Insecticide resistant has developed due to genetic variation in large insect populations. A few individuals in the original insect population are unaffected (resistant) differ from affected (susceptible) individuals either in the nature of the insecticide's target molecules in the insects or in the method the insects use to break down toxin molecules. ${ }^{(8)}$ When the insecticide is applied, individuals who are unaffected by it are those who survive to pass their genes on to following generations. Over time, a greater and greater proportion of the insect population is unaffected by the insecticide. Large number of surviving resistant insects, attempts to control insecticide resistance can indirectly cause secondary peat outbreaks that do yet more health hazards of organisms. ${ }^{(8)}$

The genetic mechanism of $C x$. quienquefasciatus, resistance to insecticides especially to organophosphates have been well characterized by esterase (Est) gene amplification.(9) Esterase gene amplification at the Ester superlocus provides organophosphate resistance in the mosquito Culex pipiens. ${ }^{(10)}$ Resistance is one of the most important factors contributing to the ineffectiveness of mosquito control programs. All the populations studied so far showed elevated $\alpha$-esterases levels in permethrin-selected populations. ${ }^{(11)}$ Insecticide resistance is expected to directly and profoundly affect the reemergence of vector born diseases ${ }^{(12)}$ and threaten disease control. In Bangladesh, there is no specific control program for Filariasis vectors. The extensive use of malathion and permithrine for dengue control and also in pest control would have indirectly contributed to pressure for selection of resistance to insecticides in Culex quienquefasciatus.

Extensive use of different insecticides i.e. the organophosphate insecticides to control the mosquito may have brought about the genetic changes in the populations of $C x$. quinquefasciatus. Many mosquito species have already developed resistance 
against a number of insecticides all over the world.(13-14) In Bangladesh, synthetic insecticides have been a major tool used in mosquito control programmes for decades and the development of resistance has led to serious problem in the overall mosquito control programme. Dhaka City is the place where insecticides are sprayed very frequently but it is not done in Sirajganj. So there is a great chance of genetic variation in the $C x$. quinquefasciatus population of the two areas in terms of insecticide resistance.

Esterase patterns vary among populations and species and are an important tool for analysis of genetic differentiation and evolutionary relationships in several insects. (15-17) Three major enzyme groups are responsible for metabolically based resistances are esterase, monooxygenase, glutathione-s-transferases (GST) to organophosphates, carbamates, organochlorines and pyrethroids. Resistance has developed to every chemical class of insecticide, including microbial drugs and insect growth regulators. (18) Insecticide resistance occurs either through changes in the targets of insecticides, typically in the nervous system or the gut, or through changes in metabolic enzymes that degrade insecticides before they reach these targets. ${ }^{(19)}$

A population is considered resistant if its response to an insecticide in detection tests drops significantly below its normal response.(20) There were 1797 cases of resistance in arthropods in $1984^{(21)}$ and by 1991, resistance to at least one insecticide had been recorded for 504 species ${ }^{(22)}$

In the light of above perspectives, present study attempts to elucidate the trend of resistance in populations of $C x$. quinquefasciatus.

\section{Materials and Methods}

Esterase patterns of adult males (M) and females (F) of Culex quinquefasciatus complex were studied using polyacrylamide slab gels following the procedure of Hames. The mosquito populations were collected as egg rafts, larvae and pupae from open drain of somewhat stagnant water in Hatirjheel, Dhaka city and Jubileebagan, Sirajganj town. Male and female adults were homogenized in $40 \mu \mathrm{l}$ of buffer solutions (0.1 M Tris-Borate-EDTA plus bromophenol blue and sucrose at $\mathrm{pH}$ 8.9). Homogenates were centrifuged at $12000 \mathrm{rpm}$ for $15 \mathrm{~min}$. After the sample application $(10 \mu \mathrm{l})$, the gels $(7.5 \%$ polyacrylamide) were submitted to a constant $120 \mathrm{~V}$, using a buffer system at room temperature $\left(\sim 25^{\circ} \mathrm{C}\right)$. The running time was $1.5 \mathrm{~h}$. Variations of this procedure, including changes in gel concentration and running time, was also tested, but the best results, i.e., clear bands for analysis, were obtained with the technique used. 
Esterase were identified in the gels following basically the technique described by Johnson et al. ${ }^{(23)}$ and Steiner and Johnson ${ }^{(24)}$, by using the $\alpha$ and $\beta$-naphthyl acetates as substrates. The technique involved gel preincubation for $15 \mathrm{~min}$, at room temperature $\left(\sim 25^{\circ} \mathrm{C}\right)$, in $104 \mathrm{ml} 0.2 \mathrm{M}$ sodium phosphates and $\alpha$ and $\beta$-naphthyl acetates at $\mathrm{pH} 8.6$, and staining reaction in the incubator $\left(37^{\circ} \mathrm{C}, 30 \mathrm{~min}\right)$ with a solution containing fast blue ruthenium red. The gels were destained during $15 \mathrm{~min}$ in a solution of methanol, glacial acetic acid, and water in a proportion of $2: 1: 8$. The electrophoretic bands of esterases resulting from staining for napthyl acetates were assigned an increased number with a decrease in mobility.

\section{Results and Discussion}

A total of eight esterase bands were detected in different adult individuals of Culex quinquefasciatus of Hatirjheel, Dhaka City and seven bands in Sirajganj Town. They were designated Est-1 to Est-7 from the less anodic to the more anodic ends of the gel (Figs. 1, $2 \&$ Table 1).

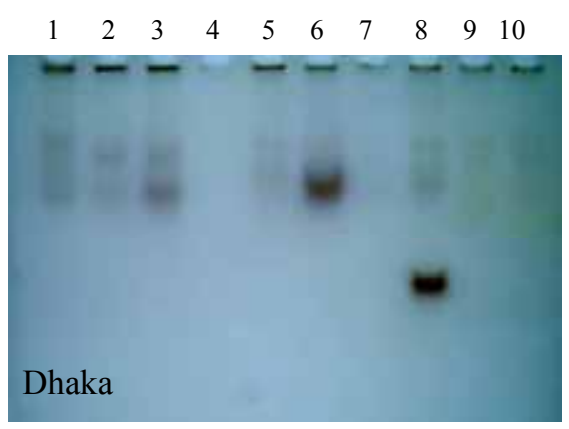

$\mathbf{A}$

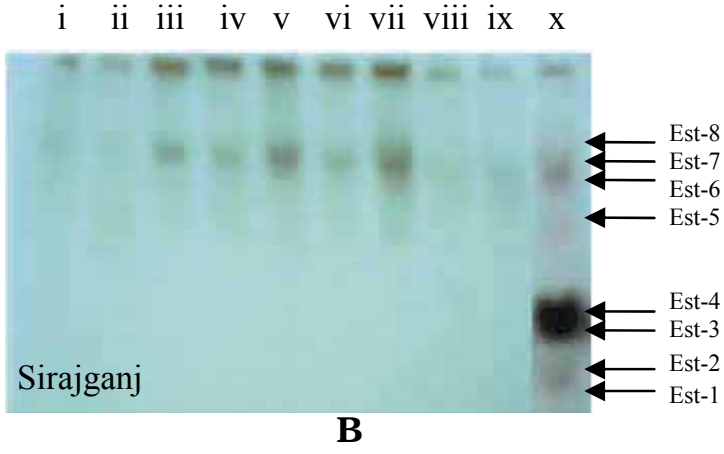

Fig.1. Esterase isozyme pattern in Culex quinquefasciatus, staining $\alpha$ and $\beta$ napthyle acetate as substrate. (A-plate indicates the population of Dhaka City and B for Sirajganj Town). Lanes 1-10 and i-x denote adult mosquitoes collected from Dhaka and Sirajganj, respectively. Arrow indicates position of esterase bands.

Est-1, the relative mobility is 3.44. Only one sample in Dhaka City and one in Sirajganj Town showed this band. Both the bands were moderately stained. Est-2 was found only in two samples of Sirajganj which are morphologically different from others. They have appeared as 50-100 fold thick reddish band which expresses $\beta$ specific nature and high insecticide resistance of that individual than others. Est-3 is the $2^{\text {nd }}$ band having the relative mobility of 1.88 . About 18 samples out of 70 of Hatirjhil, Dhaka City showed this band. But this band was not found in any samples of Sirajganj. Est-4 has the relative mobility of 1.56. In Dhaka city population, 24 mosquito samples showed this band. All most all the bands were moderately stained and two were deeply stained. In the samples of the Sirajganj, 4 showed darkly 
stained Est-4 band. The relative mobility of Est- 5 band was 1.25 and was observed in 37 samples of Dhaka City and 29 samples of Sirajganj Town. Majority showed moderately stained bands, few were faintly and darkly stained esterase bands. The most common band was Est-6. The frequency of this band was very high. Its relative mobility was 1.0. Almost all bands of Dhaka City were darkly stained whereas the bands were lightly stained in Sirajganj samples. Est-7 bands with the relative mobility 0.62 were observed in almost all samples, but they were moderately stained. The relative mobility of the Est-8 was 0.38 .

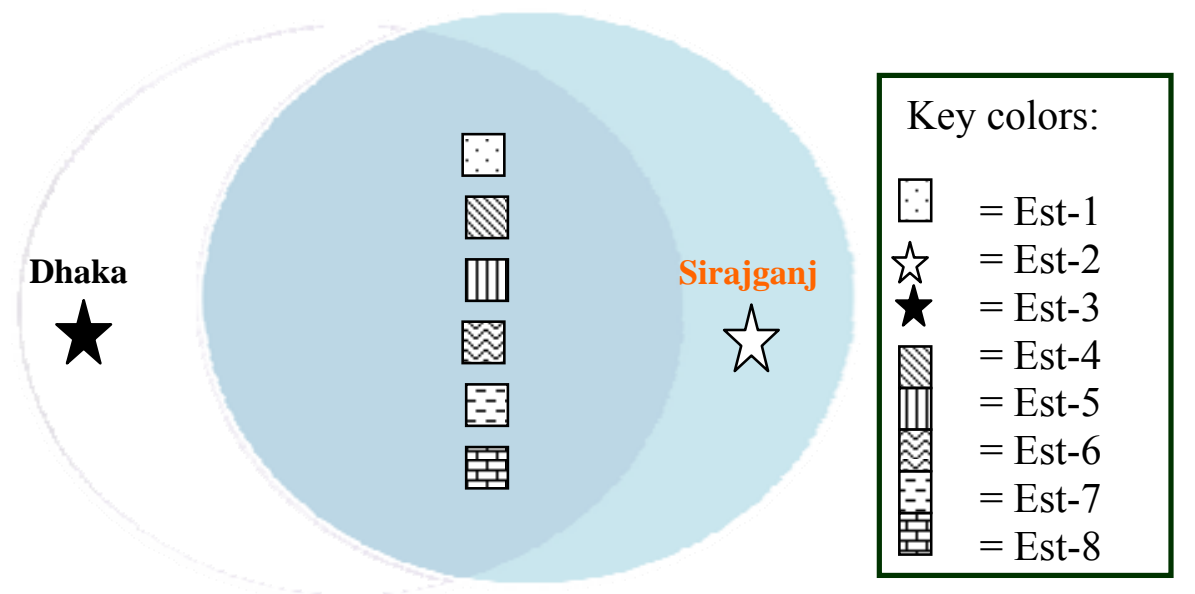

Fig. 2. Ven chart showing common and variants of esterase bands found in the population from Dhaka City and Sirajganj Town.

In the present study, the esterase bands of the samples of Hatirjheel were very deeply stained than those of the Sirajganj. The insecticides are not frequently sprayed in Sirajganj town so this may be the reason of the considerably light bands than that of Hatirjheel, Dhaka city. This indicates the more susceptibility of mosquito population of Sirajganj and was supported by our lab bioassay tools. A strong differentiation in the distribution of resistance genes high correlated with the presence or absence of organophosphate insecticide treatments on field populations of Cx. pipiens from Italy. (25) The occurrence of high resistance to malathion and DDT in two field strains of $C x$. quinquefasciatus from Kualalumpur indicates that they were under heavy pressures from organophosphorous compounds on adults through indoor and outdoor house spraying of malathion ${ }^{(26)}$ and appears to be true during the study of Dhaka and Sirajganj.

The advantages of using insecticides for mosquito control are that they can be organized within a short period of time, are effective and can produce quick results at reasonably low cost. (11) That the use on a vast and increasing scale has led to the widespread development of insecticide resistance, which has been defined as the 
development of an ability in a strain of insects to tolerate doses of toxicants which would prove lethal to the majority of individuals in a normal population of the same species. $^{(27)}$

Table 1. Esterase enzyme pattern and their frequency in percentage among adult Culex quinquefasciatus SAY population from Dhaka city and Sirajganj town (in parentheses).

\begin{tabular}{|c|c|c|c|c|c|c|c|c|}
\hline \multirow{2}{*}{$\begin{array}{l}\text { Sample } \\
\text { No. }\end{array}$} & \multicolumn{8}{|c|}{ Esterase bands } \\
\hline & Est-1 ${ }^{3.44}$ & Est-22.25 & Est- 31.88 & Est- 41.56 & Est-5 ${ }^{1.25}$ & Est- $6^{1.0}$ & Est-70.62 & Est- $8^{0.38}$ \\
\hline 1(i) & $-(-)$ & $-(-)$ & $-(-)$ & $-(-)$ & $-(++)$ & $-(+)$ & $++(++)$ & $-(-)$ \\
\hline 2(ii) & $-(-)$ & $-(-)$ & $-(-)$ & $-(-)$ & $+++(+)$ & $++(++)$ & $++(++)$ & $-(-)$ \\
\hline 3(iii) & $-(-)$ & $-(-)$ & $-(-)$ & $-(-)$ & $+++(+++)$ & $++(++)$ & $++(++)$ & $-(-)$ \\
\hline 4(iv) & $-(-)$ & $-(-)$ & $-(-)$ & $-(-)$ & $-(+++)$ & $++(-)$ & $+(++)$ & $-(-)$ \\
\hline $5(v)$ & $-(-)$ & $-(-)$ & $-(-)$ & $-(-)$ & $++(+++)$ & $++(-)$ & $++(++)$ & $-(-)$ \\
\hline 6(vi) & $-(-)$ & $-(-)$ & $-(-)$ & $-(-)$ & $-(+++)$ & $-(-)$ & $++(++)$ & $-(-)$ \\
\hline 7 (vii) & $-(-)$ & $-(-)$ & $-(-)$ & $-(-)$ & $+++(+++)$ & $+(-)$ & $++(++)$ & $-(-)$ \\
\hline 8(viii) & $-(-)$ & $-(-)$ & $-(-)$ & $-(-)$ & $-(+)$ & $++(-)$ & $++(++)$ & $-(-)$ \\
\hline 9(ix) & $-(-)$ & $-(-)$ & $-(-)$ & $-(-)$ & $+++(++)$ & $++(++)$ & $++(+)$ & $-(-)$ \\
\hline $10(\mathrm{x})$ & $-(+++)$ & $-(+++)$ & $-(-)$ & $-(-)$ & $+++(++)$ & $++(++)$ & $++(+)$ & $-(-)$ \\
\hline Frequency & $1.4(3)$ & $0(2.2)$ & $11.4(0)$ & $22.9(1.87)$ & $27.2(2.86)$ & $64.3(94.29)$ & $45.7(90)$ & $21.4(88.57)$ \\
\hline
\end{tabular}

+: Faintly stained. ++: Medium stained. +++: Deeply stained. -: Absent.

Cx. pipiens complex in the United States is composed of two subspecies, $C x$. pipiens pipiens and $C x$. pipiens quinquefasciatus, whereas intermediate forms of $C x$. p. pipiens/Cx. p. quinquefasciatus complex was also occurred. $(28,3,4,5,30,29,7)$ In China, Culex pipiens complex consists of two subspecies, Cx. pipiens quinquefasciatus and Cx. pipiens pallens that were not reproductively isolated. (30) Furthermore, in Sirajganj population two female samples out of 70 found to be produce unique, very thick ( 50-100fold) bands in Est-2, these specimens were morphologically more or less similar to other samples, alleged to be the occurrence of species complex in $C x$. quinquefasciatus.

In light of intensive use of insecticides in agriculture, however, we cannot discard the possibility that the esterase patterns of mosquitoes in Sirajganj town are being affected. This indicates a great social problem for the up coming future generations. Further investigation on $C x$. quinquefasciatus will not only help to increase our knowledge in controlling diseases like filariasis, encephalitis but also in controlling this annoying large number of pest.

\section{References}

1. Bangladesh Dengue website. <www.geocities.com/prevent_dengue>.

2. WHO (World Health Organization) 1992. Vector resistance to pesticides. Fifteenth Report of the WHO Expert Committee on Vector Biology and Control. WHO Tech. Rep. Ser. 818: $1-62$. 
3. Cheng ML, CS Hacker, SC Pryor, RE Ferrel and GB Kitto 1982. In: WWM Steiner, WJ Tabachnick, KS Rai, and S. Narang (eds.), Recent developments in the genetics of insect disease vectors. Stipes Publishing Company, Champaign, IL. The ecological genetics of Culex pipiens complex in North America, pp. 581-627.

4. Tabachnick WJ and JR Powell 1983. Genetic analysis of Culex pipiens populations in the central valley of California. Ann. Entomol. Soc. Am. 76: 715-720.

5. Pryor SC and J Daly 1991. Temporal variation in morphological and genetic characteristics within a hybrid population of Culex pipiens (Diptera: Culicidae). J. Med. Entomol. 28: $481-486$.

6. Urbanelli S, F Silverstrini, WK Reisen, E Vito and L Bullini 1997. California hybrid zone between Culex pipiens pipiens and Cx. p. quinquefasciatus revisited (Diptera: Culicidae). J. Med. Entomol. 34: 116-127.

7. Cornel AJ, RD Mcabee, J Rasgon, MA Stanich, TW Scott and M Coetzee 2003. Differences in extent of genetic introgression between sympatric Culex pipiens and Culex quinquefasciatus (Dipetra: Culicidae) in California and South Africa. J. Med. Entomol. 40: $36-51$.

8. Baumann L and P Baumann 1991. Effects of components of the Bacillus sphaericus toxin on mosquito larvae and mosquito derived from tissue culture grown cells. Current Microbiology 23: 51-57.

9. Mouchès C, P Pauplin, M Agarwal, L Lemieux, M Herzog and M Abadon 1990. Characterization of amplification core and esterase B1 gene responsible for insecticide resistance in Culex. PNAS. U S A. 87: 2574-2578.

10. Berticat C, MP Dubois, M Marquine, C Chevillon and M Raymond 2000. A molecular test to identify resistance alleles at the Ester super locus in the mosquito Culex pipiens. Pest Manag. Sc. 56: 727-731.

11. Flores AE, W Albeldano-Va'zquez, IF Salas, MH Badii, HL Becerra, GP Garcia, SL Fuentes, WG Brogdon, WC Black and B Beaty 2005. Elevated a-esterase levels associated with permethrin tolerance in Aedes aegypti (L.) from Baja California, Mexico. Pesticide Biochemistry and Physiology 82: 66-78.

12. Krogstad DJ 1996. Malaria as a reemerging disease. Epidemiol. Rev. 18: 77-89.

13. WHO (World Health Organization) 1982. Manual on Environmental Management for Mosquito Control (with special emphasis on malaria vectors). WHO, Geneva, Switzerland. $284 \mathrm{pp}$.

14. Bisset J, M Rodríguez and M Fernăndez 2006. Selection of insensitive Acetylcholinesterase as a resistance mechanism in Aedes aegypti (Diptera: Culicidae) from Santiago de Cuba. J. Med. Entomol. 43(6): 1185-1189.

15. Castiglioni-Ruiz L, MC Bicudo and CR Ceron 1997. Esterase patterns in four Brazilian populations of Hematobia irritans. Cytobios. 90: 81-94.

16. Lapenta AS, HEM C Bicudo, CR Ceron and JA Cordeiro 1998. Esterase patterns and phylogenetic relationships of species in the Drosophila buzzatii cluster. Cytobios. 96: 95-107. 
17. Nascimento AP and HEM Bicudo de C 2002. Esterase patterns and phylogenetic relationships of Drosophila species in the saltans subgroup (saltans group). Genetica (The Netherlands) 114: 41-51.

18. Brogdon WG and JC McAllister 1998. Insecticide Resistance and Vector Control. Emerging Infectious Diseases. 4: 605-613.

19. French-Constant HR 2006. Which come first: insecticides or resistance? Trends in Genetics. 23(1): 1-4.

20. Georghiou GP and RB Mellon 1983. In: G.P. Georghiou and T. Saito [eds.], Pest resistance to pesticides. Plenum Press, N Y. Pesticide resistance in time and space. pp. 1-46.

21. Georghiou GP 1986. In: Pesticide resistance: strategies and tactics for management. National Academy Press, Washington, D.C. The magnitude of the resistance problem. pp.14-43.

22. Georghiou GP and A Lagunes-Tejada 1991. The occurrence of resistance to pesticides in arthropods. An index of cases reported through 1989. FAO, Rome.

23. Johnson FM, CG Kanapi, RH Richardson, MR Wheeler and WS Stone 1966. An operational classification of Drosophila esterases for species comparison. Univ. Texas Public. 6615: 517-532.

24. Steiner WWM and WE Johnson 1973. Techniques for electrophoresis of Hawaian Drosophila.US-IBP. Island Ecosyst. Tech. Rep. 30: 1-21.

25. Silvestrini F, C Severini V, R Pardo Romi, E Matthaeis and M Raymond 1998. Population structure and dynamics of insecticide resistance genes in Culex pipiens populations from Italy. Heredity. 81: 342-348.

26. Nazni WA, HL Lee and AH Azahari 2005. Adult and larval insecticide susceptibility status of Culex quinquefasciatus (Say) mosquitoes in Kuala Lumpur, Malaysia. Trop. Biomed. 22(1): 63-68.

27. WHO (World Health Organization) 1957. Technical Report Series No. 125, 1957; Insecticides seventh report of the expert committee.

28. Barr AR 1982. In: WWM WJ Steiner, Tabachnick, KS Rai and S Narang [eds.], Recent developments in the genetics of insect disease vectors. Stipes Publ. Com. Champaign, IL. The Culex pipiens complex, pp. 551-572.

29. Crabtree MB, HM Savage and BR Miller 1997. Development of a polymerase chain reaction assay for differentiation between Culex pipiens pipiens and $C x$. $p$. quinquefasciatus (Diptera: Culicidae) in North America based on genomic differences identified by subtractivehybridization. J. Med. Entomol. 34: 532-537.

30. Zhao T and B Lu 1995. Biosystematics of Culex pipiens complex in China. Entomologia Sinica 2: 1-8. 